\title{
Oral Intragastric DMBA Administration Induces Acute Lymphocytic Leukemia and Other Tumors in Male Wistar Rats
}

\author{
Omar S El-Masry', Hussain Abdullah Alhawaj', Muaz O Fagere (D)', Amani Y Owaidah (D)', \\ Aisha Alamri (D)', Khaldoon Alsamman' \\ 'Department of Clinical Laboratory Sciences, College of Applied Medical Sciences, Imam Abdulrahman Bin Faisal University, Dammam, 3 I44I, Saudi \\ Arabia; ${ }^{2}$ Animal House Facility, Institute for Research and Medical Consultations, Imam Abdulrahman Bin Faisal University, Dammam, 3I44I, Saudi \\ Arabia \\ Correspondence: Khaldoon Alsamman, Department of Clinical Laboratory Sciences, College of Applied Medical Sciences, Imam Abdulrahman Bin \\ Faisal University, Dammam, 3I44I, Saudi Arabia, Email kmalsamman@iau.edu.sa
}

\begin{abstract}
Background: Animal models of blood cancer are important tools to study these malignancies and also screen for novel therapeutic agents. Evidence from past research on the carcinogenic properties of 7,12-dimethylbenz[a]-anthracene (DMBA) was provided by a handful of studies. However, recent literature on DMBA carcinogenic activity and the underlying mechanisms is scarce.

Objective: The aim of this study was to develop a chemical model of leukemia using DMBA. Male Wistar rats (6 weeks old) were administered $1.5 \mathrm{mg}$ of DMBA dissolved in sesame oil in biweekly doses using oral intragastric intubation.

Materials and Methods: Frequent complete blood counts and blood smear morphology assessment were used to assess the development of leukemia, while gross pathology and histopathology staining were used to evaluate malignancy development.

Results: The results showed that only $4 \%$ of rats developed acute lymphocytic leukemia. Interestingly, 36\% of the rats developed tumors (parotid tumors [24\%] and fibrosarcomas [12\%]).

Conclusion: These results suggest the pleiotropic potential of DMBA in the induction of multiple types of malignancies, including leukemia. This could be used as a model to validate therapeutic targets for leukemia and other induced malignancies.
\end{abstract}

Keywords: 7, 12-dimethylbenz[a]-anthracene, DMBA, leukemia, parotid gland, fibrosarcoma

\section{Introduction}

The need to develop an in vivo model of blood cancer is essential not only to create a disease model but also to validate newly identified therapeutic targets before moving forward into clinical trials. Therefore, attempts to induce leukemia using different leukemogenic chemicals have been made. 7,12-dimethylbenz[a]-anthracene (DMBA) is a polycyclic aromatic hydrocarbon (PAH), which is widely known as a carcinogen that initiates and promotes tumorigenesis. ${ }^{1}$ Previous studies have reported the use of DMBA as a leukemogenic chemical that induces myeloid leukemia and other types of cancers (breast and ear canal carcinomas) in Sprague-Dawley, Fischer, and Wistar rats. ${ }^{2}$ Similarly, induction of leukemia by DMBA in Long-Evans rats was also reported. ${ }^{3}$ Chemical induction of leukemia by 3-methylcholanthrene was also reviewed in the literature. ${ }^{4}$ In a similar context, a recent article ${ }^{5}$ also reported on the establishment of leukemia xenograft model by implanting patients' derived leukemic cells in immune-compromised mice and the useful use of these models to study mechanistic processes of leukemia therapies. ${ }^{5}$ In the same line, radiation-induced chronic myeloid leukemia and acute lymphocytic leukemia were also reported. ${ }^{4,6}$ It was reported that radiation-induced acute myeloid leukemia (AML) in SJL/J mouse strain mimics in its presentation human secondary AML resulting from radiation therapy of Hodgkin's lymphoma. ${ }^{4}$ Moreover, the development of animal models of mixed lineage leukemia (MLL) fusion protein (MLL-FP) acute myeloid leukemia was reported, and these models were used to validate drugs for human disease. $^{7}$ 
It was reported that the molecular mechanisms by which DMBA promoted leukemia involved N-Ras mutations as well as other chromosomal aberrations. These included duplicated locations and trisomy that were depicted in karyotypes along with some $\mathrm{Abl}$ and H-Ras rearrangements. ${ }^{8}$ The same group actively reporting on chemical induction of leukemia indicated that some DMBA-leukemic rats showed trisomy of chromosome 2 upon karyotyping and re-confirmed the N-Ras mutagenic effect of this chemical. ${ }^{9}$ Recently, it was reported that DMBA treatment induced DNA breakdown in mammary stem cells. ${ }^{10}$ In this context, the formation of apurinic/apyrimidinic sites in DNA due to treatment with DMBA was higher than that of other hydrocarbons used in the same study, indicating its DNA damaging potential. In addition, it was proposed that the formation of stable DNA adducts was probably the primary mechanism of DMBA and other PAH carcinogenesis. ${ }^{11}$ Additionally, the potential of DMBA to induce chromosomal aberrations in bone marrow was reported to be erythropoietin-dependent, suggesting the role of this hormone in the tumorigenic influence of DMBA. ${ }^{3}$ DMBA genotoxic effect is thought to be mediated by its metabolite, diol epoxide. The genotoxic effects of DMBA include mutagenesis and polyploidy and sister chromatids exchanges, which have been proved in multiple animal and in vitro models. ${ }^{12}$ Moreover, the carcinogenic effect of DMBA was promoted by other chemicals, such as tetrazine, in rat breast cancer model. ${ }^{13}$ The effect of DMBA extends to biochemical signaling, where treatment of DMBA was found to activate Fyn and Lck Src-family kinases linked to T-cell receptor, which results in activation of phospholipase $\mathrm{C}$ and mobilization of intracellular calcium. ${ }^{14}$ However, the full potential of DMBA in the rat model of leukemia has not been fully explored. The current study aimed to develop an in vivo model of leukemia to be used for validation of new therapeutic targets and to investigate the tumorigenic potential of DMBA in male Wistar rats.

\section{Materials and Methods}

\section{Ethical Approval}

The study was reviewed and approved by the IRB of Imam Abdulrahman Bin Faisal University (IRB\# 2017-03-147), and by the Animal Care Unit Committee (ACUC) at Imam Abdulrahman bin Faisal University. The project was conducted in accordance with the National Advisory Committee for Laboratory Animal Research (NACLAR) guidelines and IAU policy for the use of animals in research.

\section{Animal Models}

A total of 30 inbred male Wistar rats (6 weeks old) were obtained from the animal house facility at the Institute for Research and Medical Consultations (IRMC), Imam Abdulrahman Bin Faisal University. The animals were divided into six groups of five that were kept in separate cages, where one group was used as a control, and the other five were used for induction of leukemia using DMBA (\#D3254, Sigma-Aldrich Chemie GmbH, Taufkirchen, Germany). The animals were fed a standard diet and given access to bottled water ad libitum.

\section{Leukemia Induction}

Leukemia was induced by administering $1.5 \mathrm{mg}$ DMBA twice/week according to the dose used by Prigozhina EL. ${ }^{8,15}$ DMBA was freshly dissolved in sesame oil before administration via oral intubation. Sesame oil was administered to the corresponding control rats at the same frequency as the chemical. Rats were observed by the appointed veterinarian to identify physical signs of leukemia, such as characteristic weight loss, pale foot pads, splenomegaly, and hepatomegaly. Dose-limiting toxicities were defined as the appearance of lesions, masses or unusual physical signs, petechiae, or onset of leukemia, which was important to exclude unnecessary overdosing of DMBA during the course of treatment. In addition, the maximum treatment time was set to 8 months to avoid animal aging.

\section{Blood Collection for Complete Blood Count and Blood Smears}

The rats were anesthetized by controlled inhalation of oxygen/sevoflurane (SEVOrane, \#4456, AbbVie, Illinois, United States) mixture, and whole blood was collected from the tail vein in EDTA tubes. The blood smears were prepared immediately, air-dried, and stained using the Ral-555 staining kit (\#361550, Ral diagnostics, Montesquieu, 33650 Martillac, France) according to the manufacturer's instructions. The remaining blood samples were sent for automated 
complete blood count (CBC) and differential count analyses. White blood cell (WBC) differential count was done using a 100x oil immersion lens, and WBCs were counted until 100 cells were reached. Blood smears were evaluated by a specialized hematopathologist.

\section{Animal Dissection and Histopathology Biopsy and Tissue Fixation}

Some rats were dissected after the development of tumor masses in the parotid gland, while others were dissected after confirmation of abnormal hematology profile and enlarged viscera as a result of hepatomegaly and splenomegaly.

The rats were individually anesthetized with an oxygen-sevoflurane mixture (95-5\%) for 5 min before being stabilized in a wax tray and dissected by an appointed veterinarian. All vital organs were removed, including the brain and induced masses. Organs were washed with saline and fixed in 10\% buffered neutralized formalin (\#NBF03-500R, Tissuepro Technology, USA) overnight at room temperature. Fixed tissue was then washed thoroughly under running tap water, rinsed with distilled water, wiped off, and instantly stored at $-20^{\circ} \mathrm{C}$ until further processing.

\section{Tissue Processing and Staining}

The frozen tissues were brought to room temperature and then dissected by a histopathologist for tissue processing. The dissected tissues were packed into cassettes and embedded in paraffin wax using an automated tissue processor (Shandon Citadel 1000, Thermo Scientific, Cheshire, UK). Briefly, the tissues were incubated in two stations of formalin for 5 min as they were pre-fixed, followed by tissue dehydration through gradual concentrations of ethanol, starting with $70 \%$ ethanol (15 min), 80\% ethanol (15 min), 90\% ethanol (15 min), and finally in 100\% ethanol for $15 \mathrm{~min}$. Tissue sections were then cleared at two stations of xylene (20 min each) before being infiltrated with paraffin wax.

\section{Sections Blocking}

Wax-infiltrated sections were then transferred to a tissue-embedding center containing molten paraffin wax (TES 99, Medite Medical GmbH, Germany). The cassette was disassembled, the tissue section was properly oriented by the histopathologist, and topped with paraffin wax. The whole cassette was then transferred to a cold plate to solidify and form a block and then stored overnight in the fridge at $4^{\circ} \mathrm{C}$.

Blocks were sectioned at $5 \mathrm{~mm}$ thickness using a rotary microtome (Accu-cut ${ }^{\circledR}$ SRM $^{\mathrm{TM}}$, Sakura Finetek Europe, the Netherlands). The section ribbons were then placed in a water bath and attached onto slides. The slides were then placed on a hot plate to enhance the attachment.

\section{Section Preparation for Histological Staining}

The slides that were fixed on the hot plate following block sectioning were first dewaxed by immersing them in two successive stations of xylene (\#9990501, Shandon, Thermo Scientific, Cheshire, UK); each for 5 min. Next, the slides were hydrated in 100\% ethanol for 3 min, followed by immersion in $95 \%$ and $80 \%$ ethanol; each for 3 min, and then the slides were immersed in $70 \%$ ethanol for $3 \mathrm{~min}$. After rehydration, the slides were washed for two minutes in distilled water.

\section{Hematoxylin and Eosin Staining}

The hydrated slides were stained with Harris hematoxylin (\#6765003, Shandon, Thermo Scientific, Cheshire, UK) for 5 min and then washed for $1 \mathrm{~min}$ in distilled water. The sections were then placed under running tap water for 10 min to allow the bluing development of the stain. Slides were then washed in distilled water for $1 \mathrm{~min}$ and placed in eosin Y (\#6766008, Shandon, Thermo Scientific, Cheshire, UK) for $3 \mathrm{~min}$. Next, the slides were dehydrated in 70\% ethanol (1 $\mathrm{min}$ ), then in $80 \%$ ethanol $(1 \mathrm{~min})$, followed by $95 \%$ ethanol $(1 \mathrm{~min})$, and finally in $100 \%$ ethanol for $1 \mathrm{~min}$. The dehydrated slides were then cleared with two changes of xylene ( 2 min each), mounted with Consul-Mount (ConsulMount, \#9990440, Shandon, Thermo Scientific, Cheshire, UK), and covered with a coverslip.

\section{Masson's Trichrome Stain}

The rehydrated slides were stained with the modified Masson's trichrome stain kit for visualization of the collagenous fibers according to the manufacturer's instructions (\#MMT-M-001-50, Molequle-on, Auckland, New Zealand). Briefly, 
slides were deparaffinized and hydrated before being immersed in the Bouin's reagent (which was preheated to $56^{\circ} \mathrm{C}$ ) for $60 \mathrm{~min}$. Slides were left to cool down for 10 minutes before being rinsed in tap water until sections are clear. This was followed by another rinse in distilled water. Subsequently, slides were stained for $5 \mathrm{~min}$ in a fresh working solution of Weigert's Iron, Hematoxylin reagent (prepared by mixing equal parts of Weigert's (A) and Weigert's (B) reagents). Then, slides were rinsed in tap water for 2 min, and Biebrich Scarlet/Acid Fuchsin Solution was applied to the slides for 15 min. Next, slides were rinsed in distilled water, and the differential solution of phosphomolybdic/Phosphotungstic acid was applied to the slides for 10-15 min or until collagen red color disappears. Aniline Blue Solution was then applied to the slides for 5-10 min without rinsing. Slides were then rinsed in distilled water, and 1\% acetic acid was applied for 3-5 min. Next, slides were dehydrated in two successive changes of $95 \%$ ethanol, followed by two changes of $100 \%$ ethanol, then slides were cleared in xylene and mounted for visualization. Collagen is stained in blue, keratin and cytoplasm are red, and nuclei in blue/black.

\section{Results}

DMBA treatment resulted in the induction of more than one type of neoplasm at an average time of 6 months. The types of neoplasms and their induction percentages are summarized in Table 1. The majority of affected rats developed masses in the parotid gland, followed by sarcomas. Only one rat had a confirmed diagnosis of leukemia. The average time of induction of malignancies was 6 months, while leukemia induction was confirmed after 7 months of treatment with DMBA, which was acute lymphocytic leukemia (ALL). However, ALL was induced in a small subset of cases (4\%).

\section{Leukemia Diagnosis}

Diagnosis of leukemia was confirmed based on the gross pathological appearance of the liver and spleen, which showed characteristic organomegaly in comparison to the control rats, as shown in the gross pathology (Figure 1); hepatomegaly and splenomegaly used as hallmarks of leukemia induction. Automated $\mathrm{CBC}$ with differential also confirmed the presence of blasts and atypical lymphocytes upon treatment with DMBA, which were observed in some rats, particularly in those that developed neoplasms. These rats showed marked lymphocytosis and erythrocytosis with infiltration of nucleated red blood cells (NRBCs) into the peripheral blood and eosinophilia. Blood smears confirmed these findings; in particular, the leukemic rat blood smear revealed 53\% of lymphoblasts showing a high nuclear/cytoplasmic ratio with multiple nuclei. An interesting observation was the presence of NRBCs, atypical lymphocytes, and remarkable Rouleaux formation. In addition, one smear depicted characteristic aggregates of platelets in one rat, which showed signs of blood thinning, including pale foot pads and thin blood observed during dissection (Figure 2). Table 2 summarizes the readings of blood smears recorded in animals that developed leukemia or other masses.

\section{Gross- and Histo-Pathological Results}

As reported in Table 1, DMBA treatment resulted in not only induction of leukemia but also induced other types of malignancies that were confirmed by remarkable masses and lesions at the sites of tumor induction. Figure 1 shows the gross pathology of the organs and dissected tumor masses. The leukemic rats developed remarkable splenomegaly in comparison to the control group (Figure 1A). DMBA treatment promoted tumorigenesis in the connective tissue, resulting in an obvious induction of very large sarcomas in several other rats (Figure 1B and C). Several other rats developed masses in the parotid gland, as depicted in Figure 1D-F.

Table I Incidence of Different Types of Malignancies in DMBA-Treated Animals

\begin{tabular}{|l|c|c|c|}
\hline Number of Rats & Mass Origin & Percentage & Type \\
\hline I & Bone marrow & $4 \%$ & ALL \\
\hline 6 & Parotid gland & $24 \%$ & Non-leukemia neoplasms 36\% \\
\cline { 1 - 2 } & Connective tissue & $12 \%$ & \\
\hline
\end{tabular}

Abbreviations: ALL, acute lymphocytic leukemia; DMBA, 7,12-dimethylbenz[a]-anthracene. 
A

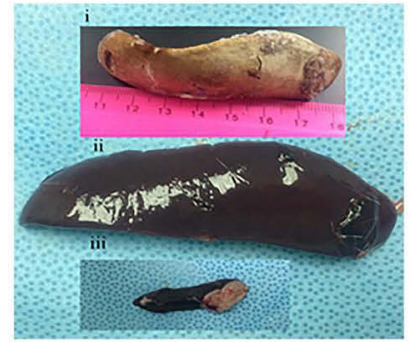

C

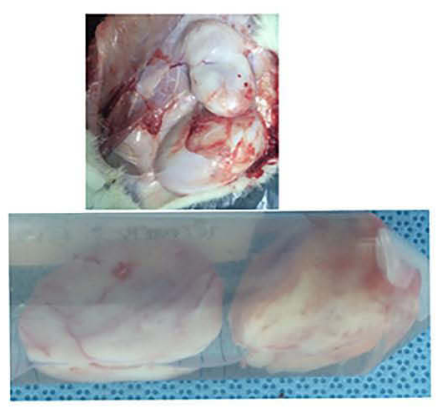

$\mathbf{E}$

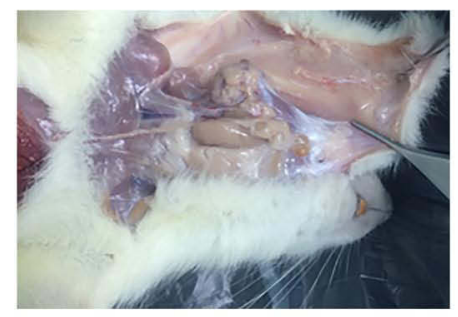

B

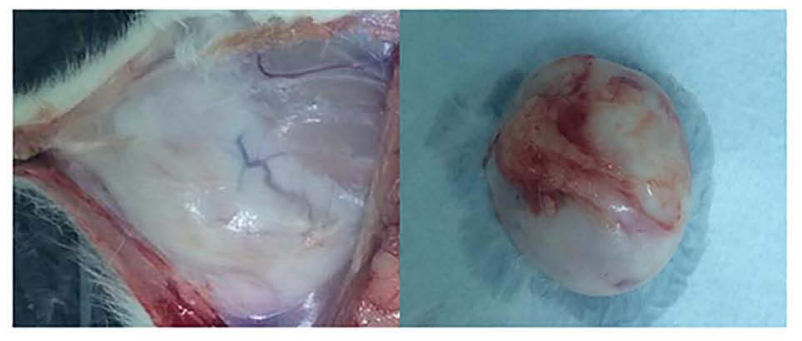

D

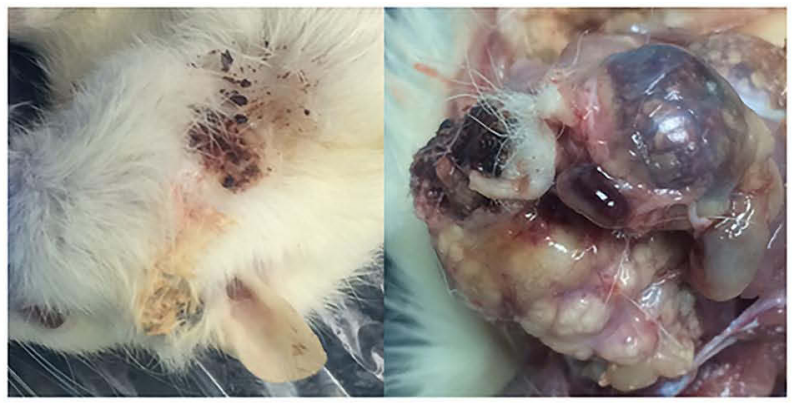

$\mathbf{F}$

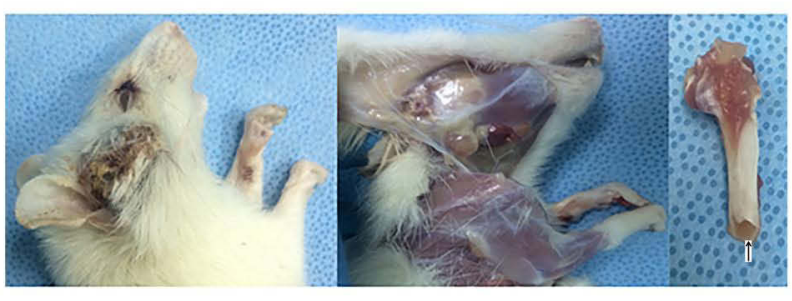

G

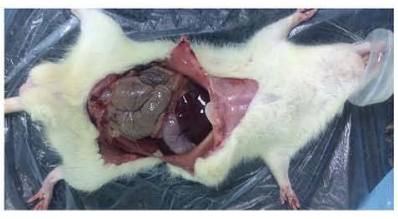

Figure I Representative gross pathology in dissected animals. The leukemic rats have remarkable splenomegaly, where the formalin-fixed spleen measured $7 \mathrm{~cm}$ (Ai), and the gross spleen (Aii) are remarkably larger than the control organ (Aiii). Representative pictures of DMBA-elicited fibrosarcomas are shown in (B and C), while representative pictures of parotid gland lesions are shown in (D-F) (white bone marrow [black arrow]). A full view of a normal control rat is shown in (G). DMBA, 7,I2dimethylbenz[a]-anthracene.

Figure 3 shows the hematoxylin and eosin and Masson's trichrome staining results. Upon histopathological staining, the enlarged spleen presented clear shrinkage of the red pulp and dissemination of the white pulp with an apparent increase in the fibrotic tissues, as indicated by Masson's trichrome staining, when compared to the control spleen. The normal control liver showed intact hexagonal parenchymal hepatocytes with homogenous distribution of chromatin in their nuclei, a landscape that was clearly lost in the enlarged liver of the leukemic rat with deposition of fibrotic tissue and inclusion of fatty vacuoles.

Normal parotid gland sections showed normal glandular epithelial tissue. Hematoxylin and eosin stained malignant parotid tissue had multiple undifferentiated malignant cells with prominent multiple nuclei in the glandular tissue and deposition of collagenous fibers as shown by the Masson's trichrome stain (Figure 3). Likewise, DMBA-induced sarcomas showed an apparent spread of malignant undifferentiated cells with multi-nuclei patterns, indicating high 
A

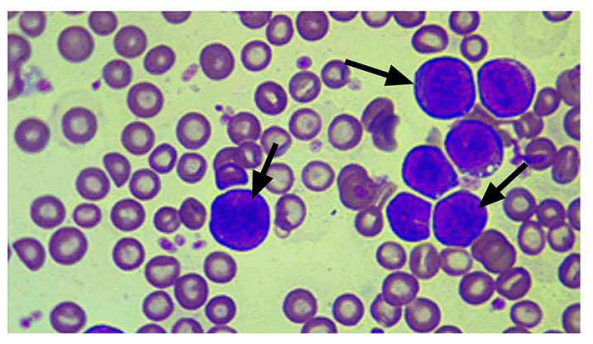

C

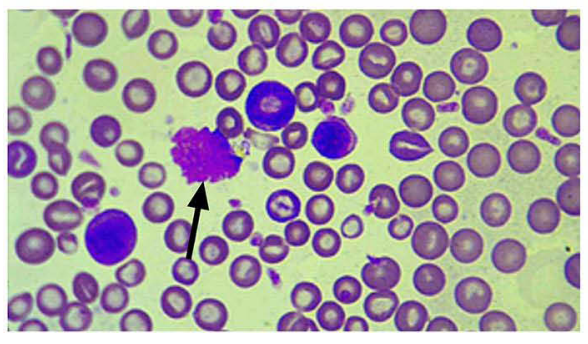

E

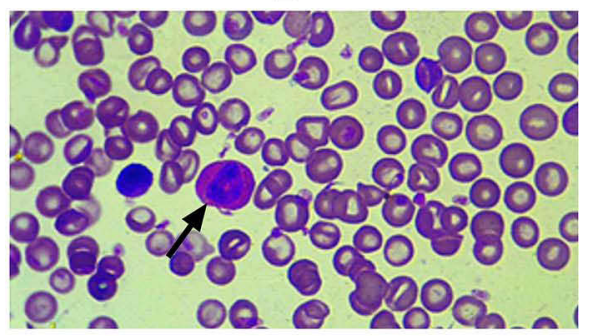

B

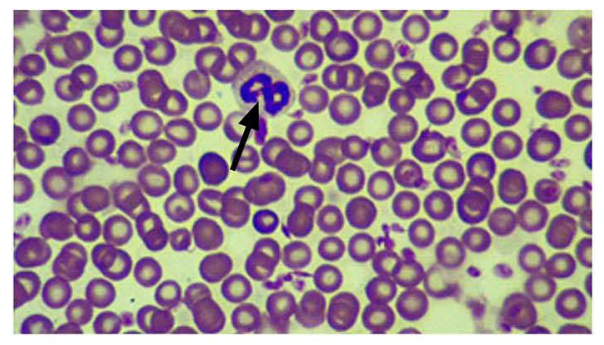

D

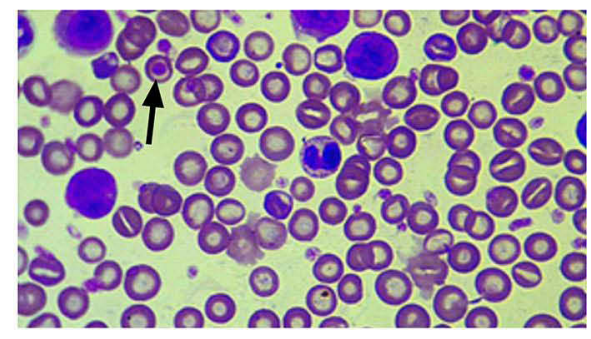

F

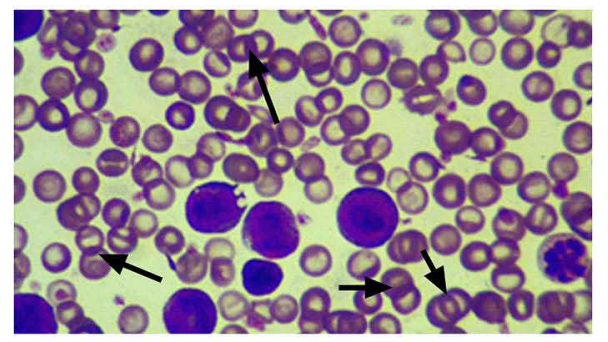

Figure 2 Representative blood smears in DMBA-treated Wistar rats. A significant number of lymphoblasts showing a large nuclear/cytoplasmic ratio and multiple nuclei (A) in the smear of the leukemic rat (black arrows point to lymphoblasts in the field with apparent high nuclear/cytoplasm ratio). A representative atypical lymphocyte is shown in another smear (black arrow; B), aggregates of platelets in a smear of one rat (black arrow; C), a representative smear showing NRBC (black arrow; D), eosinophil is shown in $\mathrm{E}$ and indicated by the black arrow, and Rouleaux stacks in F (black arrows point to multiple Rouleaux stacks in the field). DMBA, 7,I2-dimethylbenz[a]-anthracene; NRBC, nucleated red blood cells.

mitotic activity as compared to that in the normal connective tissue. Collagen and keratin fibers can be visualized as blue and red upon staining with Masson's trichrome stain.

\section{Discussion}

This study investigated the tumorigenic potential of DMBA, and in particular, explored its leukemogenic influence in male Wistar rats to create a model for the validation of possible therapeutic agents. Although the carcinogenic potential of DMBA has been reported, there is a scarcity in recent literature regarding its potential. The results of the study showed that treating male Wistar rats with biweekly doses of DMBA for an average of 6 months or more resulted in carcinogenic effects that were manifested by the induction of acute lymphocytic leukemia (ALL) (4\%), which might highlight the weak potential of DMBA to induce this type of leukemia and of parotid gland malignancy and sarcomas (36\%). ALL diagnosis was confirmed by $\mathrm{CBC}$ and blood smears, which showed infiltration of 52\% lymphoblasts in peripheral blood smears. Gross and histopathological staining confirmed malignant transformation of parotid gland tissues and sarcomas. In the context of the tumorigenic potential of DMBA, it was reported that DMBA induced mammary cancer in Wistar rats in a study that evaluated the diagnostic value of thermography in comparison to histopathology as the gold standard. ${ }^{16}$ This mammary carcinogenic effect of DMBA has also been reported by another group. ${ }^{17}$ Likewise, mammary cancer was induced in FVB mice after administration of six doses of DMBA, wherein a number of genetic aberrations 
Table 2 Readings of Blood Smears in Animals That Developed Malignancies and in Controls

\begin{tabular}{|l|c|c|c|c|c|c|c|}
\hline \multirow{2}{*}{ Group } & \multicolumn{7}{|c|}{ Average Cell Type (\%) } \\
\cline { 2 - 9 } & Lymphocytes & Neutrophils & Monocytes & Eosinophiles & Blasts & Atypical Lymphocytes & NRBCs \\
\hline Control & 40 & 53 & 5.5 & 1.5 & 0 & 0 & 0 \\
\hline Neoplastic group & 56.5 & 37.75 & 2.4 & 3.2 & 0 & 1.4 & 0 \\
\hline ALL & 30 & 10 & 1 & 0 & 53 & 1 & 5 \\
\hline
\end{tabular}

Notes: Shaded cells indicate abnormal values of laboratory parameters.

Abbreviations: ALL, acute lymphocytic leukemia; NRBC, nucleated red blood cells.

and protein post-translational modifications occurred, including overexpression of c-myc, aryl hydrocarbon receptor cyclin-D1, and hyperphosphorylation of retinoblastoma. ${ }^{18}$ In this regard, it was also reported that mammary tumors induced by DMBA showed activating mutations in Pik3ca ${ }^{\mathrm{H} 1047}$ and loss of function mutations in PTEN, which are
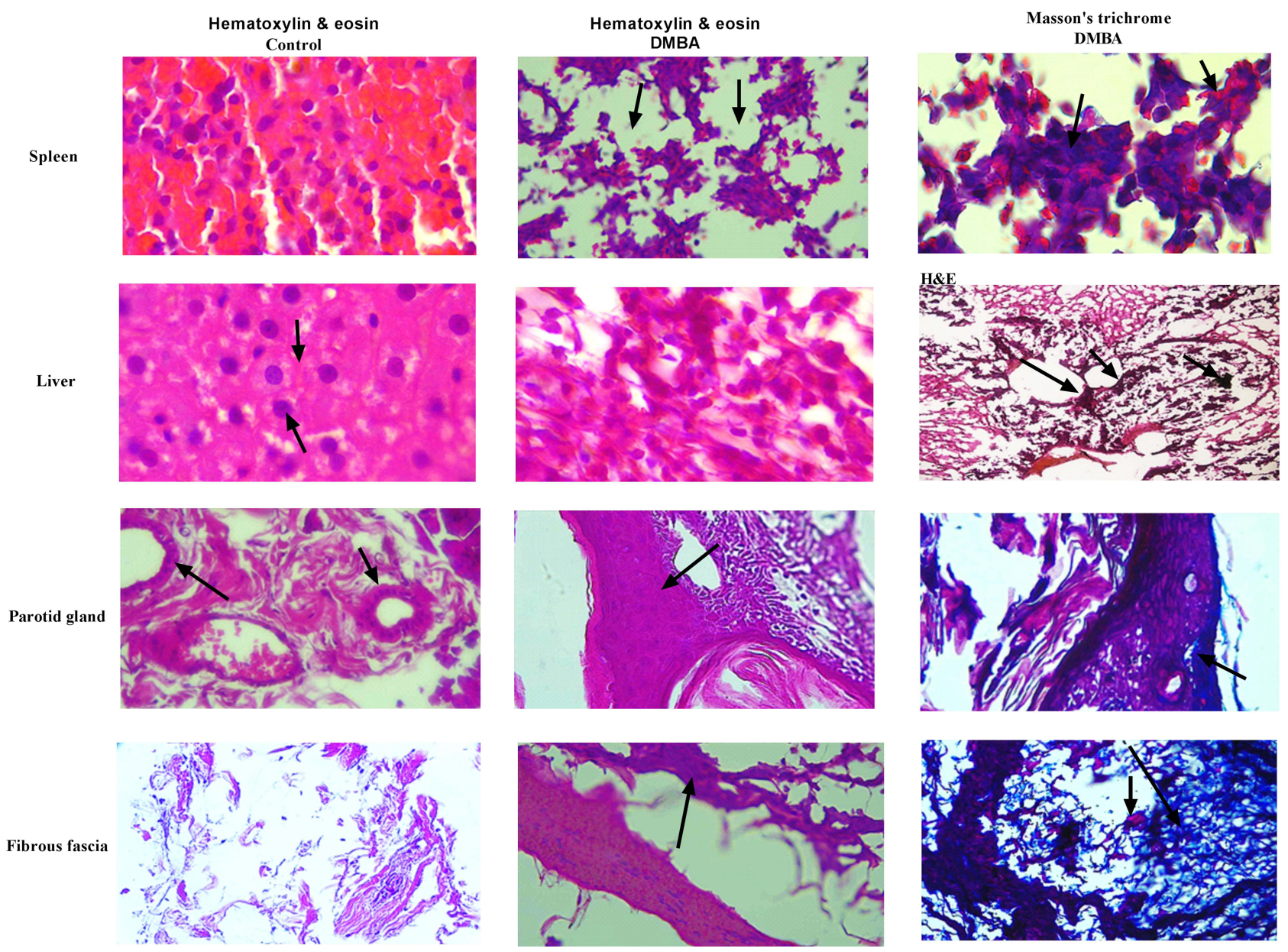

Figure 3 Hematoxylin and eosin and modified Masson's trichrome staining of control and DMBA-induced enlarged or neoplastic tissues. The control spleen shows intact red and white pulps (left). H\&E-stained enlarged spleen (middle) shows disseminated white pulp and shrinkage of the red pulp (black arrows), while Masson's trichrome shows keratin (red) and collagenous (blue) fiber deposits as indicated by the black arrows (right). The control liver (left) shows intact parenchymal hepatocytes with clear nuclear details showing homogenous chromatin distribution (black arrows), while DMBA-treated enlarged liver tissue shows a disintegrated parenchymal structure, as indicated by the loss of the hexagonal hepatocyte appearance and hyperchromatic pyknotic nucleus (middle) and fibrotic liver tissue with inclusion of fatty vacuoles indicated by black arrows (right). The control parotid tissue shows a normal circular epithelial glandular tissue (left; black arrows). H\&E-stained DMBA-treated parotid gland (middle) depicts many adjacent malignant cells (black arrow). Masson's trichrome staining (right) illustrates the blue collagenous deposits along with the malignant parotid tissue (black arrow). Fibrosarcoma developed by DMBA treatment (middle and right) shows a spread of malignant cells (middle; black arrow) as compared to the control fibrous fascia (left). Masson's trichrome staining (right) shows red and blue deposits of keratin and collagen fibers (indicated by black arrows), respectively. DMBA, 7,I2-dimethylbenz[a]anthracene; H\&E, hematoxylin and eosin. 
associated with uncontrolled activation of the PI3K/Akt pathway in human breast cancers. ${ }^{19}$ Moreover, three doses of DMBA at 4,7 , and 20 months of age induced vascular malignancies in the uterus of BALB/c female mice. ${ }^{20}$ Similarly, DMBA treatment resulted in the promotion of ovarian adenocarcinoma. ${ }^{21}$ Our results showed that double doses of DMBA administration by oral route induced sarcomas at different sites over an average period of 7 months, which was confirmed by histopathological examination and gross pathology. This was in concordance with another study ${ }^{22}$ that reported the potential of DMBA to induce sarcomas, despite the difference in dosing and time lapse for induction after treatment. According to another previous study, ${ }^{23}$ 9-10-dimethyl-1-2-benzanthracene also induced different types of sarcomas after treatment for 3-6 months. Similarly, DMBA-induced sub-maxillary salivary gland cancer was previously reported in male Wistar rats. ${ }^{24}$ These results indicate that the region encompassing the submandibular, sublingual, and parotid glands is a target for DMBA. This was in agreement with the results of the present study that suggested the pleiotropic carcinogenic potential of DMBA because it induced parotid malignancy in $24 \%$ of rats after treatment as described in the methodology section and was proved by the histopathological results. However, reports on the initiation of parotid tumors by DMBA are rare in the literature. For example, evidence from two longstanding studies ${ }^{25,26}$ documented that parotid tumors could be induced by implanting DMBA in the parotid gland of male Fischer rats for 8-14 weeks, which is different from the administration route and regimen reported in the current study. Another previous study ${ }^{27}$ reported that an in situ administration of DMBA induced submandibular gland carcinomas.

\section{Conclusions}

Our study confirmed that DMBA administration at the prescribed dose and through the adopted route and mean of administration exhibited pleiotropic carcinogenic potential, inducing ALL, carcinomas of the parotid gland, and fibrosarcoma in Wistar rats. These results consolidated the findings reported in a few longstanding studies and highlighted the tumorigenic potential of the chemical in parotid glands through oral administration rather than local implantation of the drug, which was supported by histopathological staining and examinations. In addition, our study recommends that in order to induce a leukemic model, a large number of animals should be used because the percentage of incidence is quite low at only $4 \%$.

\section{Abbreviations}

DMBA, dimethylbenz[a]-anthracene; ALL, acute lymphocytic leukemia.

\section{Acknowledgment}

The authors would like to express their sincere gratitude to the Deanship of Scientific Research at Imam Abdulrahman Bin Faisal University for funding this work.

\section{Author Contributions}

All authors made substantial contributions to conception and design, acquisition of data, analysis and interpretation of data; took part in drafting the article or revising it critically for important intellectual content; agreed to submit to the current journal; gave final approval for the version to be published; and agreed to be accountable for all aspects of the work.

\section{Funding}

This work was supported by the Deanship of Scientific Research at Imam Abdulrahman Bin Faisal University (project number: 2017-CAMS-099).

\section{Disclosure}

The authors declare they have no conflicts of interest to disclose that are relevant to this study. 


\section{References}

1. Iversen $\mathrm{OH}$. A course of very small doses of DMBA, each of them allegedly with no promoting potency, acts with clear synergistic effect as a strong promoter of DMBA-initiated mouse skin carcinogenesis. A comparison of the tumorigenic and carcinogenic effects of DMBA (7,12-dimethylbenz-alpha-anthracene) and TPA (12-O-tetradecanoyl-phorbol-13-acetate) used as initiators and promoters in classical two-stage experimental protocols. APMIS Suppl. 1994;41:1-38.

2. Pollard M, Kajima M. Leukemia induced by 7,12-dimethylbenz[a]anthracene in germfree Rats. JNCI. 1967;39(1):135-141.

3. Sugiyama T. Role of erythropoietin in 7,12-dimethylbenz(a) anthracene induction of acute chromosome aberration and leukemia in the rat. Proc Natl Acad Sci USA. 1971;68(11):2761-2764. doi:10.1073/pnas.68.11.2761

4. Almosailleakh M, Schwaller J. Murine models of acute myeloid leukaemia. Int J Mol Sci. 2019;20(2):453. doi:10.3390/ijms20020453

5. Yang X, Yuan YP, Chen YJ. [Advances of research on application of leukemia xenotransplantation model-review]. Zhongguo shi yan xue ye xue za zhi. 2019;27(4):1348-1352. Chinese. doi:10.19746/j.cnki.issn.1009-2137.2019.04.060

6. Finch SC. Radiation-induced leukemia: lessons from history. Best Pract Res Clin Haematol. 2007;20(1):109-118. doi:10.1016/j.beha.2006.10.009

7. Milne TA. Mouse models of MLL leukemia: recapitulating the human disease. Blood. 2017;129(16):2217-2223. doi:10.1182/blood-2016-10691428

8. Sugiyama T, Osaka M, Koami K, Maeda S, Ueda N. 7,12-DMBA-induced rat leukemia: a review with insights into future research. Leuk Res. 2003;26:1053-1068. doi:10.1016/S0145-2126(02)00045-0

9. Sugiyama T, Osaka M. 7, 12-DMBA-induced rat leukemia as a model of chemical carcinogenesis. Proc Japan Soc Animal Mod Human Dis. 2005;21:37-44. doi:10.1538/expanim1992.21.37

10. Ledet MM, Oswald M, Anderson R, Van de Walle GR. Differential signaling pathway activation in 7,12-dimethylbenz[a] anthracene (DMBA)-treated mammary stem/progenitor cells from species with varying mammary cancer incidence. Oncotarget. 2018;9(67):32761-32774. doi:10.18632/oncotarget.25988

11. Melendez-Colon VJ, Luch A, Seidel A, Baird WM. Cancer initiation by polycyclic aromatic hydrocarbons results from formation of stable DNA adducts rather than apurinic sites. Carcinogenesis. 1999;20(10):1885-1891. doi:10.1093/carcin/20.10.1885

12. Balakrishnan S, Menon VP, Manoharan S, Rajalingam K. Antigenotoxic effect of ferulic acid in 7,12-dimethyl benz(a)-anthracene (DMBA) induced genotoxicity. Afr J Tradit Complement Altern Med. 2007;5(1):32-38. doi:10.4314/ajtcam.v5i1.31253

13. Zingue S, Mindang ELN, Awounfack FC, et al. Oral administration of tartrazine (E102) accelerates the incidence and the development of 7,12-dimethylbenz(a) anthracene (DMBA)-induced breast cancer in rats. BMC Complement Med Ther. 2021;21(1):303. doi:10.1186/s12906-02103490-0

14. Archuleta MM, Schieven GL, Ledbetter JA, Deanin GG, Burchiel SW. 7,12-dimethylbenz[a]anthracene activates protein-tyrosine kinases Fyn and Lck in the HPB-ALL human T-cell line and increases tyrosine phosphorylation of phospholipase C-gamma 1, formation of inositol 1,4,5-trisphosphate, and mobilization of intracellular calcium. Proc Natl Acad Sci USA. 1993;90(13):6105-6109. doi:10.1073/pnas.90.13.6105

15. EL Prigozhina. Induction of leukosis in rats with dimethylbenzanthracene and its transplantation. Vopr Onkol. 1962;8:64-70.

16. Angeline Kirubha SP, Anburajan M, Venkataraman B, Akila R, Sharath D, Raj B. Evaluation of mammary cancer in 7,12-dimethylbenz(a) anthracene-induced Wister rats by asymmetrical temperature distribution analysis using thermography: a comparison with serum CEA levels and histopathology. J Biomed Biotechnol. 2012;2012:786417. doi:10.1155/2012/786417

17. Alessandra-Perini J, Perini JA, Rodrigues-Baptista KC, et al. Euterpe oleracea extract inhibits tumorigenesis effect of the chemical carcinogen DMBA in breast experimental cancer. BMC Complement Altern Med. 2018;18(1):116. doi:10.1186/s12906-018-2183-z

18. Currier N, Solomon SE, Demicco EG, et al. Oncogenic signaling pathways activated in DMBA-induced mouse mammary tumors. Toxicol Pathol. 2005;33(6):726-737. doi:10.1080/01926230500352226

19. Abba MC, Zhong Y, Lee J, et al. DMBA induced mouse mammary tumors display high incidence of activating Pik3caH1047 and loss of function Pten mutations. Oncotarget. 2016;7(39):64289-64299. doi:10.18632/oncotarget.11733

20. Tsubura A, Senzaki H, Okada H, Morii S. DMBA-induced uterine vascular tumors in BALB/c mice: ovary-dependent carcinogenic response. Anticancer Res. 1991;11(1):187-191.

21. Crist KA, Zhang Z, You M, et al. Characterization of rat ovarian adenocarcinomas developed in response to direct instillation of 7,12-dimethylbenz[a]anthracene (DMBA) coated suture. Carcinogenesis. 2005;26(5):951-957. doi:10.1093/carcin/bgi039

22. Walentinsson A, Levan G. Ras gene mutations in 7,12-dimethylbenz[a]anthracene (DMBA)-induced rat sarcomas. Cancer Lett. 2001;166(1):47-53. doi:10.1016/S0304-3835(01)00388-3

23. De Santis E, Rosa MA, Oransky M, Sanguinetti C. Experimentally induced synovial sarcoma. Int Orthop. 1981;5(1):37-41. doi:10.1007/ BF00286097

24. Ziu M, Giasuddin AS, Mohammad AR. The effect of garlic oil (allium sativum) on DMBA induced salivary gland tumorigenesis in rat. $J$ Islam Stud. 1994; 7(3):189-192.

25. el-Mofty S. Spontaneous regression of induced parotid gland tumors in rats. Oral Surg Oral Med Oral Pathol. 1978;45(3):431-440. doi:10.1016/ 0030-4220(78)90529-7

26. Sela J, Azachi C, Levij IS, Ulmansky M. Fibrosarcoma or squamous cell carcinoma of rat parotid after instillation of DMBA into the duct. $J$ Dent Res. 1974;53(6):00220345740530063601. doi:10.1177/00220345740530063601

27. Barka T. Effects of isoproterenol on mammary gland tumors induced by N-nitroso-N-methylurea and salivary gland tumors induced by 7,12-dimethylbenz[a]anthracene. J Natl Cancer Inst. 1982;69(5):1115-1120. 


\section{Publish your work in this journal}

The Journal of Experimental Pharmacology is an international, peer-reviewed, open access journal publishing original research, reports, reviews and commentaries on all areas of laboratory and experimental pharmacology. The manuscript management system is completely online and includes a very quick and fair peer-review system. Visit http://www.dovepress.com/testimonials.php to read real quotes from published authors.

Submit your manuscript here: https://www.dovepress.com/journal-of-experimental-pharmacology-journal 